\title{
Visual selection mediated by location: Selecting successive visual objects
}

\author{
KYLE R. CAVE \\ Vanderbilt University, Nashville, Tennessee \\ and \\ HAL PASHLER \\ University of California, San Diego, La Jolla, California
}

\begin{abstract}
In these experiments, each stimulus consists of a series of frames, each containing a target digit of one color and a distractor digit of another color. The task is to name the highest digit of the target color. Subjects make fewer errors when successive targets appear at the same location than when they appear at different locations, apparently because they select target objects by using a mechanism that is based on location. When successive targets appear at the same location, there is no need to "move" the selection mechanism to a new location, leaving more time to identify the stimuli. These experiments show that location-based selection is used even though selection by color would be more direct. They also demonstrate a method of measuring location-based selection that can be applied to a variety of visual tasks. Further experiments reveal that although location-based selection is used to identify a digit in the presence of a digit distractor, it is not used to identify a digit in the presence of a letter distractor, suggesting that this selection mechanism is not used in this situation to prevent interference among the basic features making up letters and digits, but to inhibit responses associated with the distractors.
\end{abstract}

Over the past 10 or 15 years, many studies have probed the nature of the "attentional spotlight." Researchers have tried to determine its shape (C. W. Eriksen \& St. James, 1986; Podgorny \& Shepard, 1983), measure its size (Cave \& Kosslyn, 1989; Egeth, 1977; C. W. Eriksen \& St. James, 1986; LaBerge, 1983; Larsen \& Bundesen, 1978), assess the sharpness of its edge (Downing, 1988; C. W. Eriksen \& St. James, 1986; LaBerge \& Brown, 1989), clock its speed as it moves (Murphy \& C. W. Eriksen, 1987; Remington \& Pierce, 1984; Sagi \& Julesz, 1985; Tsal, 1983), detect its presence as it passes over locations (Murphy \& C. W. Eriksen, 1987; Shulman, Remington, \& McLean, 1979), and test its ability to split into multiple spotlights (C. W. Eriksen \& Yeh, 1985; Posner, Snyder, \& Davidson, 1980). These studies are all based on one central idea - that visual selection is based on location, or, in other words, that information originating

This research was performed while K.R.C. was a fellow at the Center for Human Information Processing, University of California, San Diego. Thanks to Alann Lopes for programming and maintaining the computers used in these experiments; to Marlisa Anderson, Angelique Bruny, and Hanh Tran for conducting the experiments; and to Steve Luck, Jeff Schall, Claus Bundesen, and two anonymous reviewers for knowledgeable advice. This work was supported by National Institute of Mental Health Training Grant MH-14268, awarded to the Center for Human Information Processing, University of California, San Diego, and by NCA2-225 and NCA2-414 from NASA, N00014-91-J-1735 from ONR, and 1-R29-MH45584-01 from NIMH, awarded to H.P. Correspondence should be addressed to K. R. Cave, Vanderbilt University, Department of Psychology, 301 Wilson Hall, Nashville, TN 37240 (e-mail: cavekr@ctrvax.vanderbilt.edu). from one particular location or region in the visual field is chosen for special processing, while information from all other locations is excluded. Despite the importance of this spatial attention assumption to many current accounts of visual selection, it has not been tested very thoroughly.

Much of the impetus for the idea of selection by location came from spatial cuing experiments, which showed that subjects respond faster to a stimulus when they know its location before it appears (C. W. Eriksen \& Hoffman, 1974; Posner, Nissen, \& Ogden, 1978; Posner et al., 1980). In general, cuing studies have provided important data concerning visual attention. However, these data by themselves are unlikely to present a complete picture of visual selection. First, the instructions in these studies suggest that location is an important property. The subject is led to expect each stimulus at a particular location and may decide to select that location and to exclude others, even if other methods of selection are available or if selection is not required in this task. Second, in most of these studies, stimuli have appeared on a more or less blank display, with no distractors to filter out. Visual selection is probably far more critical when distractors are present (Shiu \& Pashler, 1994). We are likely to see different aspects of the performance of visual selection if we test it under varying degrees of load, and the most interesting properties of selection may only appear under conditions in which targets must be selected and distractors must be excluded.

A complete picture of visual selection requires experiments that include distractors and that test whether lo- 
cation affects selection even when location is irrelevant to the task and selection by location is not suggested by the instructions. If selection is based on location, and if we assume that it takes time and effort to shift attention from one location to another, tasks involving multiple stimuli should be easier when the stimuli are at or near the same location. In one relevant experiment by Hoffman and Nelson (1981), subjects searched for target letters among a rapidly changing array of four letters (similar to displays used by Schneider \& Shiffrin, 1977). At the same time, they also watched for a small U-shaped figure to appear near one of the letters and determined its orientation when it did. On trials in which they correctly identified which target letter appeared, they were more likely to correctly report the orientation of the U-shaped figure if it was positioned near the target letter. When they were wrong about the letter, they were more likely to be right about the orientation of the U-shaped figure if it was not near the target letter. These results indicate that two objects can be processed more effectively when they are close together. This pattern would be expected if some sort of facilitation were applied to a particular region at any given time. Whenever the area around one visual object is chosen for facilitation, other objects near it should benefit, and objects far from it should suffer. Hoffman, Nelson, and Houck (1983) provided additional evidence for this hypothesis. ${ }^{1}$

These experiments show that, at least under some circumstances, two targets are easier to identify when they both appear at or near the same location. If selection is based on location, it might also be harder to prevent interference from distractors that are near a target. C. W. Eriksen and Hoffman (1972) used configurations of letters to show that distractors near a target (within about $1^{\circ}$ ) interfere with naming the target, whereas distractors farther away do not. This interference disappears if the target location is cued at least $150 \mathrm{msec}$ in advance. C. W. Eriksen and Hoffman (1973) and B. A. Eriksen and C. W. Eriksen (1974) demonstrated that this interference occurred at the response stage, because the interference was much greater when the nearby distractors were associated with a different response. (In a later experiment, Gatti \& Egeth, 1978, found Stroop interference from distractors as far away as $5^{\circ}$ from target.)

Further evidence for selection by location can be found in the errors that subjects make when they select the wrong stimulus in a multielement display. Snyder (1972) found that when subjects misreported the shape of a stimulus selected by the criterion of color, they were likely to report the properties of a neighboring stimulus. In similar studies by Tsàl and Lavie (1988, 1993), the stimuli most likely to be reported by subjects immediately after a search task were those near the search target. Tsal and Lavie (1993) showed that even when subjects could ignore all aspects of a stimulus other than its color, they were still faster to respond to a second stimulus when it was located near the first, suggesting that they selected the target by location even for simple color detection. Additional evidence comes from studies by LaBerge and Brown $(1986,1989)$ showing that allocation of attention to certain areas for one task can affect the processing of other stimuli appearing at the same locations.

Nissen (1985) demonstrated a special role of location in selection by using a different approach. On the basis of Treisman's feature integration theory (Treisman \& Gelade, 1980), she hypothesized that accessing information about any visual property of a particular object would first require determining the location of that object. The stimuli for her experiments consisted of four shapes of four different colors positioned at the corners of an imaginary diamond. When subjects were given the location and asked to report both color and shape, their accuracy for these two dimensions was independent, as Nissen expected. She predicted different results for using color to find shape and location, however, since it should be impossible to find the shape of the red object without first finding its location. Using conditional probabilities, she made quantitative predictions about the percentage of trials in which subjects should make location errors, shape errors, and errors of both types together. The data fit her predictions well (although Bundesen, 1991, offers a different interpretation).

Each of these experiments suggests that location plays some role in visual selection. However, some of them rely on rather indirect demonstrations, and none of them reveals whether selection is based on location in all circumstances. For instance, when Hoffman and Nelson's (1981) subjects searched for a target among characters in an array, they may have had to rely on some type of selection to isolate each character for identification. With no other property such as color or size differentiating the characters, it would be reasonable for them to select by location. Similarly, when Tsal and Lavie's (1993) subjects were trying to report as many characters as possible from a display of eight or nine, they also may have been unable to identify all the characters at once. With no other basis for selection, they may simply have chosen to select by location, starting arbitrarily at the location nearest the previous stimulus. Tsal and Lavie (1993) eliminated this problem in Experiment 3, in which subjects were explicitly instructed to report only the letter at a particular location in the second task. Still, they were faster to report the selected letter when it was near the target for the previous task, which was relevant only in color, and not location. Thus they apparently selected the color stimulus by location. Nevertheless, preparing to select by location in the second task may have caused subjects to select the location of the color target in the first task. Tsal and Lavie (1993) addressed this alternative explanation in their final experiment. Subjects no longer reported the letter designated by location. Instead, only two letters were displayed in each trial, and subjects responded if one was an $F$. Again, responses were faster for letters near the previous target.

Taken together, these last two experiments provide good evidence that subjects selected by location even 
when the task did not require or suggest location selection. One alternative, however, must still be considered. Experiment 4 once again introduced an element of search that had been present in Experiments 1 and 2 . One of the two letters was either P or B, and the other was either $F$ or $\mathrm{H}$. Subjects responded only when an $F$ was present. With only two letters present, the need for selection was probably much less than it was with the larger displays in Experiments 1 and 2. Nevertheless, subjects had to search for an $\mathrm{F}$ between two letters that had very similar shapes. Therefore subjects may still have found it necessary to select each letter individually to identify it. The two digits had different colors, so subjects could select by color rather than location; but the colors were assigned randomly, so selecting by color in this experiment would be just as arbitrary as selecting by location. With no other basis for selection, they may have chosen to select by location, starting with the one near the previous stimulus. Thus, none of these experiments tests whether selection is based on location even when all targets are marked by color or some other simple visual property that makes location-based selection completely unnecessary.

In one relevant experiment, conducted by Broadbent (1977), subjects reported one colored word embedded in a series of successively presented black words. The words alternated between two different locations. When subjects reported the wrong word, they were more likely to report a word that appeared at the same location as that of the target word than to report one that occurred immediately before or after the target but at a different location. In other words, spatial proximity played a more important role than temporal proximity.

We set out to perform our own, more thorough, test, under circumstances in which selection by location was not explicitly required to separate target from distractors. If visual selection is based on location, and if shifting attention from one location to another requires a certain amount of effort and time, then processing a series of visual stimuli should be easier when they all appear at the same location. Furthermore, as long as the processing of these stimuli requires attention, this advantage for same-location stimuli should hold even if location is not directly relevant to the task. In the experiments reported here, this possibility was explored by using tasks in which the locations of target and distractor stimuli were irrelevant to the response. If performance is better when all target stimuli appear successively at the same location, we will conclude that a location-based selection mechanism is at work. Of course, such evidence would not support the conclusion that all selection in this task is location-based; there might be other mechanisms simultaneously selecting by other factors.

\section{EXPERIMENT 1 Selection by Color}

In each trial of this experiment, subjects viewed a series of colored digits. In each frame of the series, two digits appeared, one to the left and one to the right of a fixation cross. One of the digits in each frame was red, and the other was green. One group of subjects was instructed to attend to the green digits and to ignore the red digits, while a second group of subjects received instructions with the color roles switched. At the end of each trial, the subject reported the highest digit among those of the target color in the series of frames for that trial. In order to be sure of the correct response in the highest digit task, the subject must have identified all of the digits in the series with the target color. Thus this task differed from that used in many visual search experiments, in which the subject knows a single target in advance, and may not need to identify the other display elements.

Each subject received a random mixture of two types of trials. In a same trial, the target digits all appeared at the same position. In an alternating trial, the position of the target digit alternated from one frame to the next. If attention is allocated by position, and if there is some cost in shifting attention to a new position, we would expect performance to drop in the alternating condition relative to the same condition.

We have performed many different experiments of this type, varying the number of frames in each trial. In this experiment, each trial will have only two frames, and only $150 \mathrm{msec}$ will pass between the appearance of the first digits and the disappearance of the last. This fast display will rule out any possibility that subjects might move their eyes to the location of the first target after the presentation of the first frame.

\section{Method}

Subjects. Twenty students from the University of California, San Diego, participated in the red-target version of this experiment, and 10 participated in the green-target version. All were fulfilling a course requirement. None knew the purpose of the experiment or the expected results beforehand. Most subjects finished in less than an hour.

Apparatus. The apparatus consisted of IBM PC and compatible microcomputers equipped with Paradise VGA+ graphics cards and NEC Multisync or Multisync 2a monitors. Subjects' responses were entered on the standard keyboard.

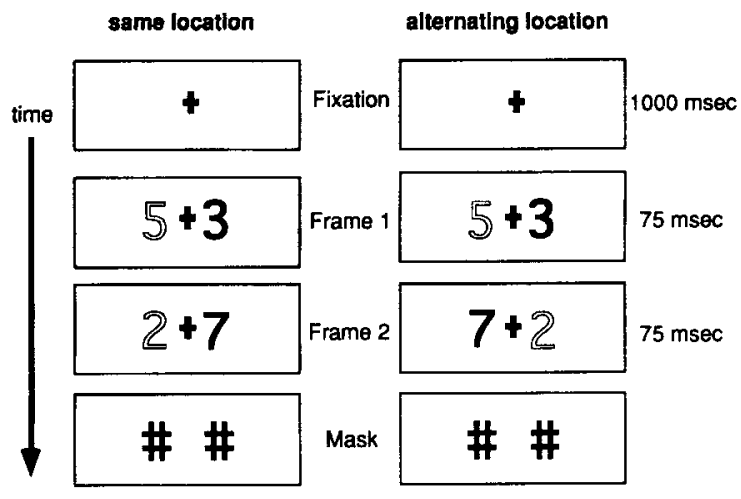

Figure 1. The sequence of displays in a single trial from Experiment 1. (Light digits represent green, and dark digits represent red.) 
Stimuli. Each trial included a series of two frames presented successively, followed by a mask. Each frame consisted of a fixation cross at the center of the screen, and two digits, one on each side of the cross, as shown in Figure 1. Thus each frame included one digit to be attended and one to be ignored. The distance from the center of one character to the center of another was $3 \mathrm{~cm}$, and each digit was $2.3 \mathrm{~cm}$ high and $1 \mathrm{~cm}$ wide. The fixation cross was $1.6 \mathrm{~cm}$ high and $.8 \mathrm{~cm}$ wide. The subject was approximately $60 \mathrm{~cm}$ from the screen. The background was white, and the fixation cross was black. In each frame, one digit was red and the other was green. The mask at the end of each trial consisted of two black "\#" characters, about the same size as the digits, and positioned at the same locations occupied by the digits in the previous frames.

For each subject, half the trials were same trials, with both target digits appearing on one side and both distractor digits on the other. Of these trials, half had targets on the left and half had targets on the right. The remaining half of the trials were alternating trials, in which the target digit appeared on different sides in the two frames. Half of the alternating trials started with a target digit on the left and half started with a target on the right.

Procedure. The sequence of displays for a single trial is illustrated in Figure 1. Each trial began with the presentation of the fixation cross. This was the subject's cue to fixate on the cross and prepare for the trial. After $1,000 \mathrm{msec}$, the two stimulus frames appeared, each for $75 \mathrm{msec}$. In this experiment and in all that follow it, the presentation of the first frame was synchronized with the video refresh. The second frame was followed by the mask, which remained visible until the subject responded. The two distractor digits were chosen randomly from the digits 1 through 9 . The two target digits were also chosen randomly, but with the constraint that the digit 9 never appear before the last frame. (If subjects saw a 9 before the last frame, they would not need to process the later digits.) Also, no digit appeared more than once in a trial, either as a target or as a distractor. At the end of the trial, subjects reported the higher of the target digits by typing the appropriate key on the keyboard. Subjects were encouraged to take as long as they needed to respond. If the response was incorrect, a tone sounded immediately, lasting $300 \mathrm{msec}$. A timed delay was added before each trial to ensure a constant 450 -msec intertrial interval, including the time for the error tone. Each subject received 672 trials, presented in 14 blocks of 48 trials each. The subjects were encouraged to rest between blocks. Each subject began with a single block of 48 practice trials. More practice was provided if the subject requested it or if the experimenter thought it was necessary.

\section{Results}

In this experiment, we purposefully did not match shades of red and green for brightness, because we were more interested in providing an effective cue for attention allocation than in comparing the effects of brightness and hue. The red stimuli used in this experiment may have been more salient in some way than the green stimuli, and thus might have interfered more with other stimuli appearing at the same position. For green subjects in the alternating condition, each target digit was either preceded or followed by a red digit, whereas in the same condition, each target was only preceded or followed by green digits. If red digits were more effective masks than green digits, targets would be more effectively masked in the alternating condition. For red subjects, targets would be more effectively masked in the same condition. Therefore, we must analyze data from the two subject groups separately to be sure that any effect was due to spatial attention and not to masking.

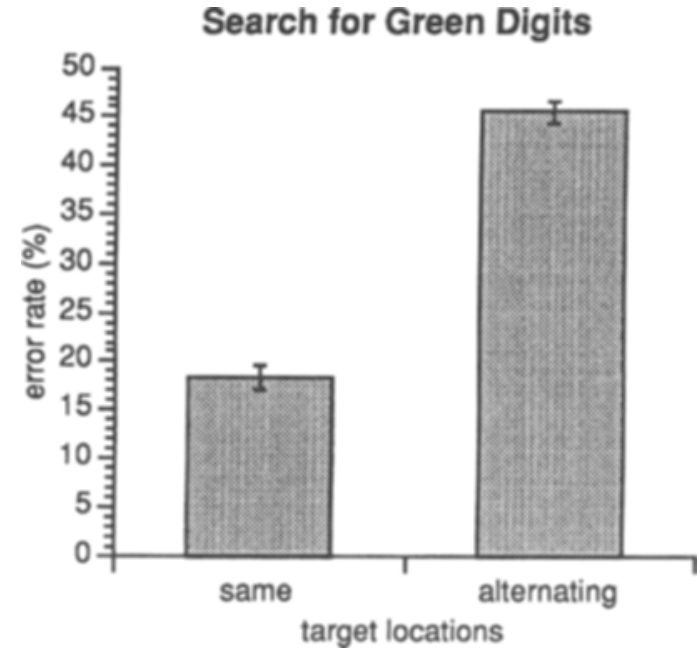

Figure 2. Error rates from Experiment 1 searches for green targets. ${ }^{2}$

Error rates for each group of subjects were subjected to repeated measures analyses of variance (ANOVAs) with same versus alternating trial and left versus right starting position as factors. Subjects searching for green targets made many more errors when the target switched positions from one frame to the next $[F(1,19)=258.02$, $p<.001]$. (See Figure 2.) In this case, there was no significant advantage for trials beginning with targets on the left or right $(F<1)$. There were $32 \%$ errors for starting on the left and $31 \%$ for starting on the right. There was no interaction between the two factors $(F<1)$.

The pattern from red-target subjects was different, however. With the target and distractor colors switched, there was no significant difference between the number of errors in the same and alternating conditions $[F(1,9)=1.3, p>.25]$. (See Figure 3.) Perhaps the red stimuli were indeed more effective at masking other

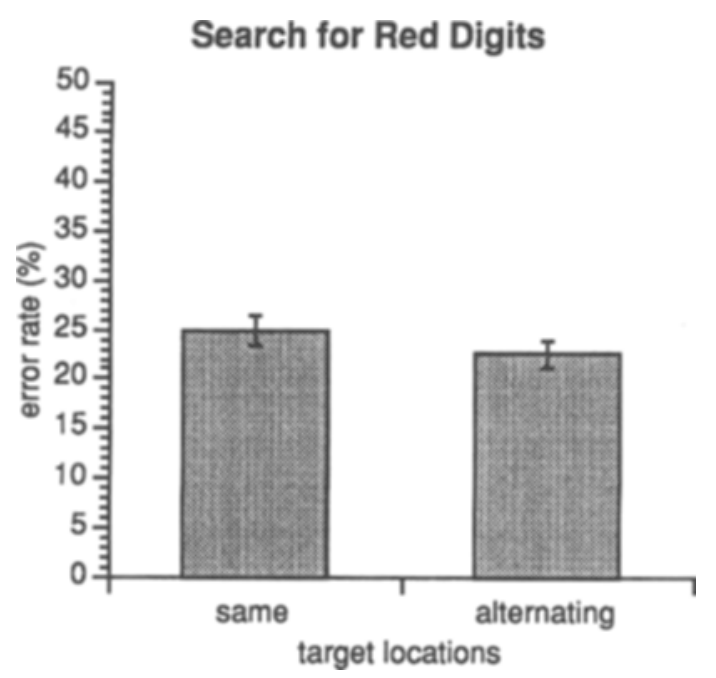

Figure 3. Error rates from Experiment 1 searches for red targets. 
stimuli appearing at the same position. In the same condition, each red target was subject to masking from the other red target, whereas in the alternating condition each red target was only subject to masking from a green distractor

The ANOVA showed no hint of an advantage for either the left or the right starting position $[F(1,9)=$ $1.9, p>.2]$, with $24 \%$ errors for starting on the left and $23 \%$ for starting on the right. There was no interaction between starting position and same versus alternating condition $(F<1)$.

\section{Discussion}

In this task, position of the digits was irrelevant to the correct response. If subjects nonetheless selected target digits by location, their accuracy should have been higher if both of the target elements appeared at the same position. Subjects searching for green digits exhibited such an advantage for same trials over alternating trials, but the lack of a significant difference in the red subjects casts doubt on the role of location-based attention in this experiment. It does not, however, rule it out. The same masking effect that might have been producing a same condition advantage in the green subjects could have been obscuring an attentional same condition advantage in the red subjects. Same trials might have been easier than alternating trials for red subjects because no attention shift was required between Frames 1 and 2, but also more difficult because of extra interference from having two red targets appear at the same location. The next experiment was an attempt to remove the masking advantage and determine whether any attentional effect would persist.

\section{EXPERIMENT 2 Mask After Each Frame}

As in Experiment 1, each trial in this experiment consisted of two frames, with two digits each. However, this time each frame was followed by a black mask identical to the mask presented at the end of each trial in the previous experiment. This mask, which consisted of a black "\#" pattern at each of the two digit locations, should thoroughly mask each of the digits, regardless of whether it was preceded or followed by a red or green digit. With masking being equal in same and alternating conditions, we could now attribute differences between these conditions to attention shifts rather than masking differences.

In Experiment 2, half the subjects attended to red digits, and half attended to green digits. We will examine the results of each group separately, and of both groups together.

\section{Method}

Subjects. Seventeen subjects participated in this experiment. Thirteen were students from the University of California, San Diego, who were fulfilling a course requirement, and 4 were paid for their participation. None knew the purpose of the experiment

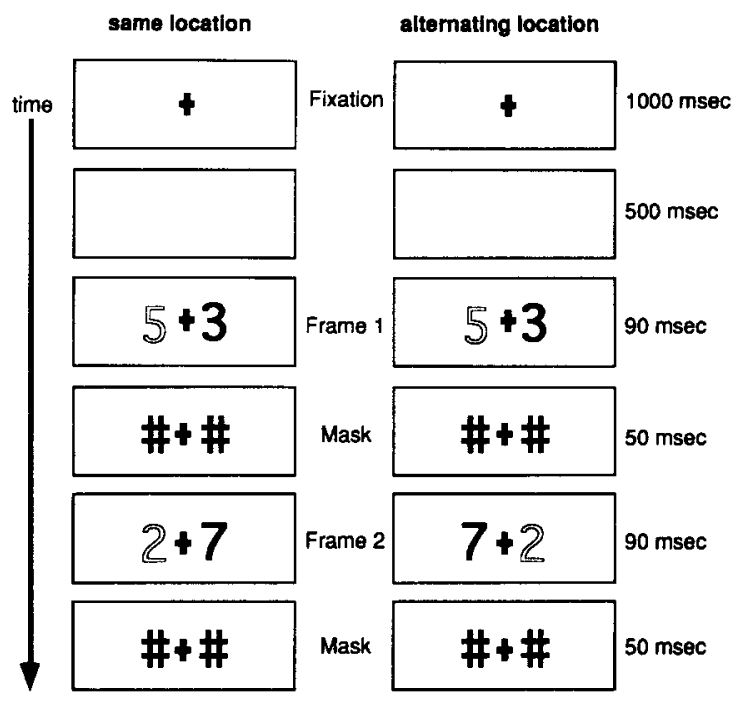

Figure 4. The sequence of displays in a single trial from Experiment 2.

or the expected results beforehand, and none had participated in Experiment 1 . The subjects usually finished in less than an hour.

Stimuli and Procedure. In each trial, the mask appeared for $50 \mathrm{msec}$ after each frame, as shown in Figure 4 . Because the mask made identification of the digits more difficult, the exposure times for the digit frames were increased to $90 \mathrm{msec}$. Nine of the subjects attended to red and ignored green, and the remaining 8 had the color roles reversed. As in Experiment 1, each subject completed 14 blocks of 48 trials each.

The time between the appearance of the first digit pair and the disappearance of the second digit pair was $230 \mathrm{msec}$. Although this amount of time might allow the initiation and execution of a saccadic eye movement, it is unlikely that subjects used saccades in this experiment. Because the subjects did not know the target locations in advance, preparation for a saccade could not begin until after the first digit pair appeared. The eyes would not actually move for approximately $180 \mathrm{msec}$ after that, long after the first digit pair had disappeared. If the saccade were completed at that time, then on half the trials it would actually move the eyes away from the target. On trials in which the saccade did foveate the second target, no more than $30 \mathrm{msec}$ of foveal processing would be possible before the mask appeared, and at least $20 \mathrm{msec}$ of processing time would have been lost as the eyes were moving. Therefore, any subjects who attempted to saccade would quickly find that it was more effective to keep their eyes fixed on the cross throughout each trial. This fact, coupled with the strong admonitions from the experimenter to keep the eyes fixed, should have prevented saccades in Experiment 2.

\section{Results and Discussion}

The error rates from all subjects were included in a single ANOVA with starting position and trial type (same vs. alternating) as factors. Error rates were $8 \%$ lower in the same condition than in the alternating condition $[F(1,16)=30.7, p<.001]$. (See Figure 5.) Thus, when masking was controlled, performance still dropped when more attention shifts were required. Starting position had no effect $(F<1)$, and there was no interaction $(F=1.5, p>.2)$. We also separated the data into two 


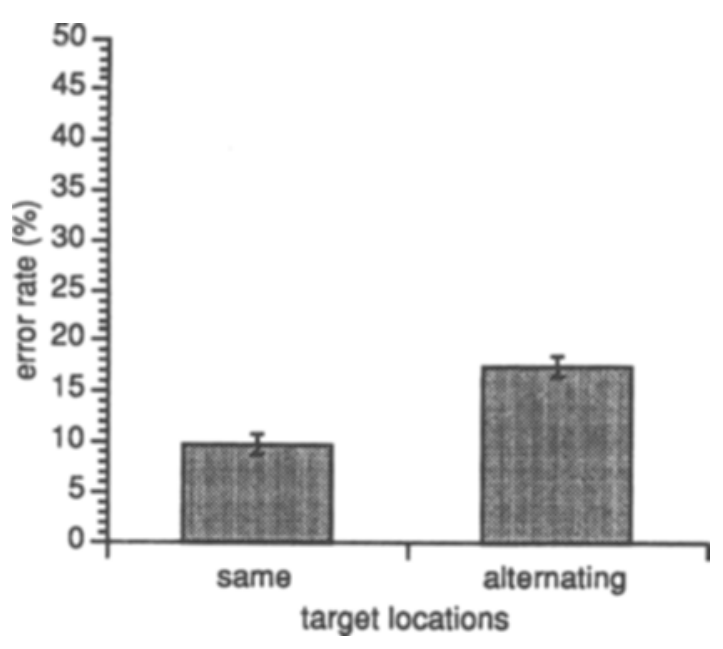

Figure 5. Error rates from Experiment 2.

groups by target color and analyzed each separately. In each group, the advantage for same trials was significant $[F(1,7)=12.5, p=.01$, for green targets, and $F(1,8)=$ $16.6, p<.01$, for red targets].

Once again, subjects made more errors when target location switched from trial to trial, just as the green subjects did in Experiment 1. In the previous experiment, some of this effect was probably due to the less effective masking from the green digits. In this experiment, however, each digit should have been thoroughly masked by the black "\#," producing the same degree of masking in the same and alternating conditions. When the masking effect was eliminated, there were still more errors in the alternating condition. Therefore, at least part of the effect measured in Experiment 1 was due to some other factor, presumably the shifting of attention from one location to another. For the red subjects in Experiment 1 , the attentional effect was eliminated by the masking effect.

A particular location can be selected by facilitating the processing of information from that location, by inhibiting the processing of information from other locations, or by both. Determining whether selection is implemented by facilitation or inhibition from experiments such as this is very difficult. In much of the discussion of the following experiments, location-based selection will be described as resulting in either facilitation of target locations or inhibition of distractor locations, but these descriptions merely refer to the relative effects of attention on different locations, and not to how attention is implemented.

In Experiment 1, the disadvantage for the alternating condition might have been due to some perceptual difficulty stemming from the changing colors at each location. Such an explanation seems less likely for Experiment 2 , because both the same and the alternating conditions had a black mask appearing before and after each digit. Thus both conditions had abrupt color changes.
One factor that must be considered in using multipleframe displays with multiple targets is the "attentional blink" described by Broadbent and Broadbent (1987) and Raymond, Shapiro, and Arnell (1992). In those studies, the presence of one target in the sequence of displays impaired the ability to detect a second target appearing as much as $500 \mathrm{msec}$ after the first. The inhibition in the attentional blink did not take effect immediately after the first target's appearance, but built over time. If there was an attentional blink in the present experiments that followed the same time course as that measured by Raymond et al., the second target would have appeared before the inhibition had become very strong. Even if an attentional blink was exerting a major effect on performance in the present experiments, however, the conclusions would remain the same. Broadbent and Broadbent and Raymond et al. did not test whether the inhibition applied to stimuli at all locations or only to the target location. If it applied to all, it would apply equally in the same and alternating conditions, with no net effect on the result. If it is location-specific, it would actually be working against the effect demonstrated in the present experiments, by raising the error rate only in the same condition. If a location-specific attentional blink was affecting these results, the advantage for a target appearing at a selected location is actually larger than the results suggest.

Another possibility is that the attentional blink is just one aspect of a location-specific inhibition that can also occur concurrently with a target to block distractors at other locations. If so, the attentional blink is just part of the location-specific selection mechanism that these experiments investigate.

\section{EXPERIMENT 3 Letter Distractors}

Experiment 2 demonstrated how comparing performance in same and alternating conditions can serve as a measure of location-based visual selection. This method makes it possible to explore what sorts of visual processing tasks elicit selection by location, and what stimulus properties can cause information from a location to be selected. In Experiment 3, we tested whether selection by location would be used when targets were defined by alphanumeric category rather than by color. If the category of a stimulus is determined early in processing, attention might be directed to the location of a character with the correct category to help in its final identification.

In Experiment 3, subjects viewed two digits and two letters in each trial. They ignored the letters and reported the higher digit. The color of the characters was irrelevant to the correct response. If in this experiment we should find the same advantage for the same condition as that found in Experiment 2, we would know that subjects can allocate spatial attention according to alphanumeric category, as they do according to color. If not, 
then either category information cannot direct spatial selection, or the different type of distractors makes spatial selection unnecessary.

\section{Method}

There were three different variations of this experiment, as described below.

Subjects. Thirty-one subjects participated in Version A of this experiment, 12 in Version B, and 20 in Version C. All were UCSD students who were fulfilling a course requirement. None knew the purpose of the experiment or the expected results beforehand, and none had participated in the previous experiments. Most subjects finished in less than an hour.

Stimuli and Procedure. The displays in all three versions of this experiment included two frames with one digit and one letter in each. Digits were chosen as they were in the previous experiments. Letters were chosen randomly from the set of all 26 letters with replacement. In Version $\mathrm{A}$, both the digits and the letters were black. Each frame was visible for $50 \mathrm{msec}$. As in Experiment 1 , there was no mask between the first and second frames, and the mask after the second frame remained visible until the subject's response. Version B was more like Experiments 1 and 2 in that each character was green or red. The color was assigned randomly to each character, however, so that color provided no information about which character was the target (digit) and which was the distractor (letter) in each frame. In this version, a mask appeared for $50 \mathrm{msec}$ between the two frames, and the mask after the second frame remained visible until the response. In Version C, red and green colors were also assigned randomly to the letters and digits, but with the constraint that one character in each frame be red and the other be green. In this version, each frame was visible for $90 \mathrm{msec}$, and each was followed by a 50 -msec mask. All three versions consisted of 14 blocks of 48 trials each.

\section{Results}

The error rates from Version A are shown on the left side of Figure 6, and those from the other two versions are on the left side of Figure 7. The error rates from each of the three versions were subjected to separate ANOVAs with starting position and trial type (same vs. alternating) as factors. For all three versions, the error rate remained the same, whether both targets appeared in the same location or not [for Version A, $F(1,30)=2.5, p>$ .1 ; for Version $\mathrm{B}, F(1,11)<1.0$; for Version $\mathrm{C}, F(1,19)=$ $1.4, p>.25]$. The error rate also remained constant regardless of whether the first digit appeared to the left or to the right [for Version $\mathrm{A}, F(1,30)<1.0$; for Version $\mathrm{B}$, $F(1,11)=2.5, p>.1$; for Version $C, F(1,19)=2.9, p>.1]$.

The interaction between trial type and starting position showed a surprising and inconsistent pattern across the three versions of this experiment. Version A showed no hint of an interaction $[F(1,30)<1]$. In the other two versions, error rates were lower when the digit in the second frame was on the left side of the display [for Version $\mathrm{B}, F(1,11)=6.9, p<.05$; for Version $\mathrm{C}, F(1,19)=$ $20.7, p<.001]$. What this pattern reflects is unclear, but in both cases, fewer errors occurred when the last target digit in the sequence was on the left.

Note that the exposure times in Version $C$ were exactly those used in Experiment 2 with digit distractors. If the more accurate responses in Experiment 2's same condition had been due to saccades, there should have been a similar advantage in Experiment 3, Version C. The lack of any effect here indicates that our assumption about fixation in Experiment 2 was correct.

\section{Discussion}

Whatever the cause of the interaction, all three versions of this experiment showed that when targets and distractors were differentiated only by alphanumeric category, and not by color, the relative location of the two targets did not affect accuracy. Subjects apparently did not use selection by location in this case, because their performance was no worse when location shifts were necessary. This result might suggest that the locationbased selection mechanism can be directed by simple visual properties such as color, but not by higher level properties such as alphanumeric category. By the time a character has been sufficiently processed to determine its category, there may be nothing to gain by subjecting
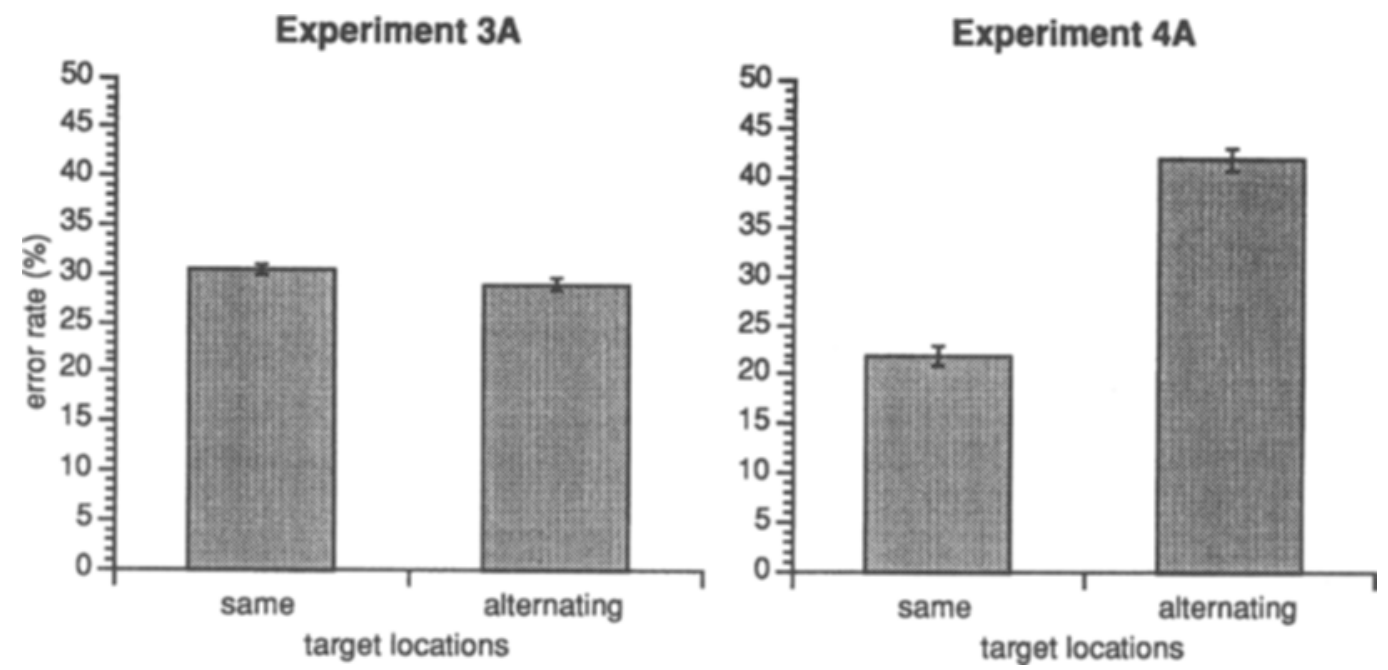

Figure 6. Results from Version $A$ of Experiments 3 and 4. 

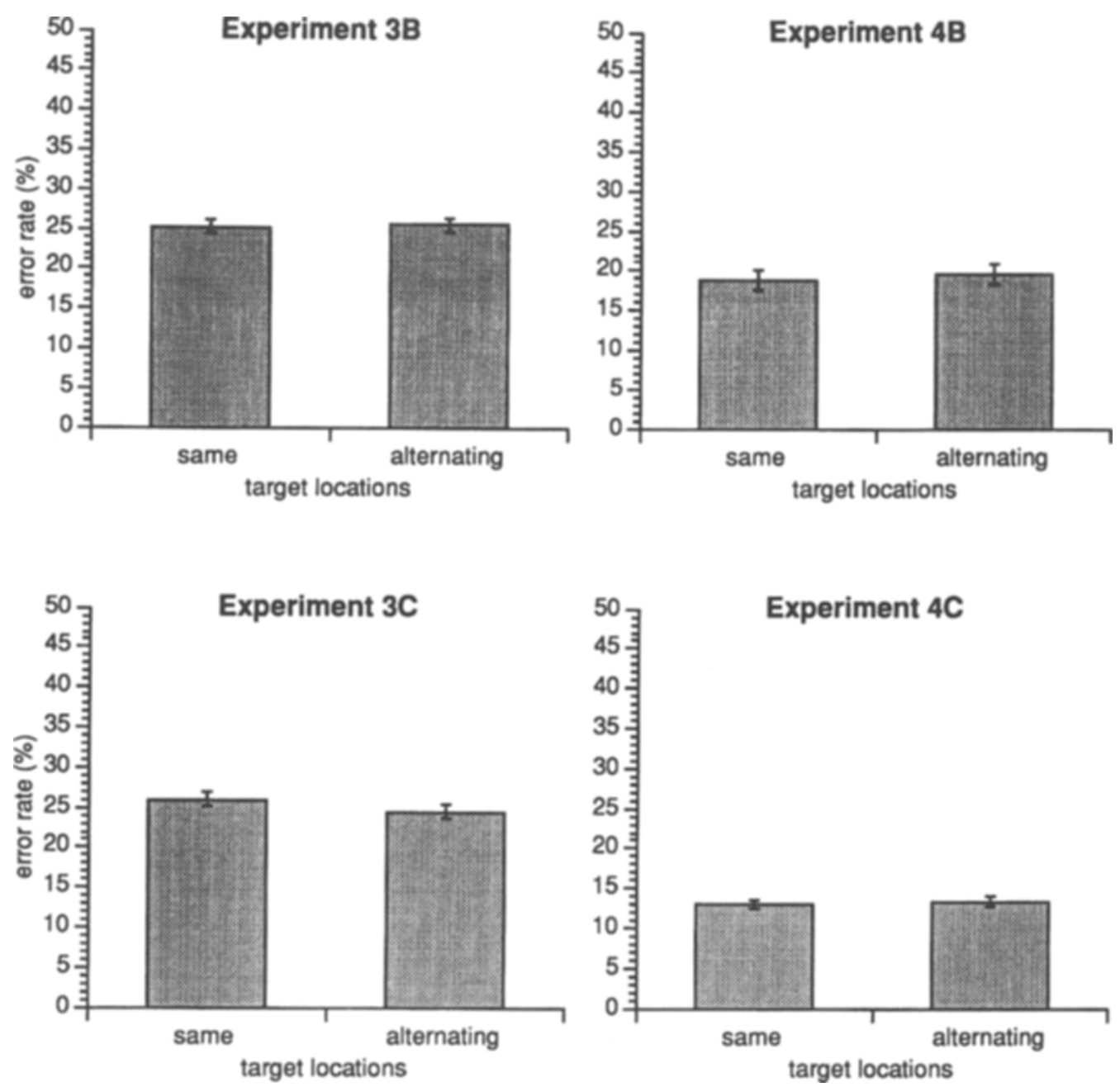

Figure 7. Results from Experiments 3 and 4, Versions $B$ and $C$.

it to location-based facilitation or inhibition. However, the next experiment will indicate that selection by location might not have been necessary in this particular task at all.

\section{EXPERIMENT 4}

\section{Letter Distractors With Color Information}

Selection by location might be so helpful to visual identification that subjects will use it whenever they have the opportunity. They might have been denied that opportunity in Experiment 3, because they could not identify the alphanumeric category of each stimulus quickly enough to direct selection. On the other hand, spatial selection may not have been as useful in this task as it was in Experiment 2. The distractors in Experiment 2 interfered with identification of the target, even though they were a different color. This interference could have occurred at a number of different processing levels. For instance, if the target was identified by detecting the set of features that it included, the presence of other features at other locations might have interfered. This feature-level interference is predicted by a number of current attentional theories, including feature integration theory (Treisman, 1988; Treisman \& Gelade, 1980) and guided search (Cave \& Wolfe, 1990; Wolfe, Cave, \& Franzel, 1989).

Experiment 3 raises another possibility. The targets in that experiment were defined by alphanumeric category, and subjects did not use location-based selection but could still perform the task very well. Perhaps there is a detector for every possible digit that responds independently of location, and each of these detectors tends to elicit a response, whether the digit that stimulates it is the target or a distractor. In this case, selection is necessary in order to prevent interference at the response level, rather than at the feature level.

In Experiment 4, the targets were digits of one color, and the distractors were letters of another color. Both color and category information were available for finding target locations. If letter distractors interfere with the identification of digits enough to make the selection process worthwhile, this experiment should show better performance in the same condition, as in Experiment 2. 
We tried a variety of different versions of this experiment, matching the different versions of Experiment 3.

\begin{abstract}
Method
Subjects. Twenty-six subjects participated in Version A of Experiment 4, 12 in Version B, and 15 in Version C. All were UCSD students. None knew the purpose of the experiment or the expected results beforehand, and none had participated in the previous experiments. Most subjects finished in less than an hour.

Stimuli and Procedure. The displays in all three versions of this experiment had two frames per trial, each with one digit and one letter. Digits and letters were chosen as they were in the previous experiment. Version A was similar to Experiment 3, Version A, with $50 \mathrm{msec} /$ frame and a single mask at the end. Digits were green and letters were red. Versions B and C both had a 50msec mask between the two frames to equate masking across the two conditions. In both versions, approximately half the subjects searched for red digits among green letters, and the other half searched for green digits among red letters. Version B corresponded to Experiment 3, Version B, with $75 \mathrm{msec} /$ frame and a final mask that remained until the response. Version $C$ corresponded to Experiment 3, Version $C$, with $90 \mathrm{msec} /$ frame and a final mask of $50 \mathrm{msec}$. All three versions consisted of 14 blocks of 48 trials each.
\end{abstract}

\section{Results}

The error rates from Version A were subjected to an ANOVA with starting position and trial type as factors. Error rates were significantly lower when both targets appeared at the same location $[F(1,25)=175.2, p<$ $.001]$. This pattern does not necessarily indicate selection by location, however, because this experiment has no mask between the two frames, allowing the same type of masking differences as was seen in Experiment 1. A comparison of the alternating conditions in Version A of Experiments 3 and 4, shown in Figure 6, makes it clear that the green targets are masked more by red characters at the same location than by green. In Experiment 3, Version A, digits and letters were all black, whereas in Experiment 4, Version A, digits were green and letters were red. Even with the color information contributing to the selection of targets, there were more errors in the alternating condition of Version A of Experiment 4 than in that of Version A, Experiment 3.

Versions $B$ and $C$ included masks after each frame and thus gave a more accurate indication of the use of location-based selection in these tasks. These data are presented in Figure 7. The data from each of these experiments were analyzed in separate ANOVAs with starting position, trial type, and target color as factors. These analyses showed a pattern like that found in Experiment 3, Versions B-C. Error rates were about the same, whether the two targets in each trial shared the same location or not $(F<1.0$ in both cases). Even though subjects now had color information to direct location-based selection, they apparently identified the targets without selecting their locations.

In none of the three versions was there any significant effect of starting position [for Version A, $F(1,25)<1$; for Version $\mathrm{B}, F(1,10)=2.9, p>.1$; for Version $\mathrm{C}$, $F(1,13)<1]$. There was no interaction between trial type and starting position in Version $\mathrm{A}[F(1,25)<1]$. Ver- sion $\mathrm{C}$ showed the same advantage for trials with the second-frame target on the left that was seen in Experiment 3 , Versions B-C $[F(1,13)=9.4, p=.01]$. A similar trend was present in Experiment 4, Version $B$, although it did not reach significance $[F(1,10)=3.0, p>.1]$.

\section{Discussion}

Subjects in Experiment 4 enjoyed a potential advantage over those in Experiment 3, in that they could use color information to differentiate targets and distractors. Nevertheless, the results from Experiment 4, Versions B-C, indicate that subjects did not rely upon selection by location, as they did when color information was available in Experiment 2. Apparently, when digits are being sought and letters are present in the display, the letters do not interfere with digit identification enough to make location-based selection worth the effort. Selection by location may still be necessary if a larger number of distractors is present.

Although subjects did not use spatial attention in Experiment 4, error rates were generally lower than in Experiment 3 , in which color information was not available. The difference between Versions $C$ of Experiments 3 and 4 was confirmed with an ANOVA $[F(1,33)=$ $10.1, p<.01]$. Although subjects were not selecting by location, they may have had some ability to select directly by color, strengthening signals from objects of the target color and attenuating those from objects of the distractor color. If selection by color is possible, it must be fairly limited, or selection by location would not have been used in any of these experiments. (See Tsal \& Lavie, 1993, for further evidence of selection by color.) Perhaps the letter distractors in Experiment 4 interfered with the digit targets enough to elicit color selection, but not enough to elicit location selection, which may take more effort, and may cause problems on alternating trials. In Experiment 3, any interference from letter distractors would not be controlled by either color or location selection, because of the lack of color cues designating targets. The uncontrolled interference in Experiment 3 could explain why the error rate in Version $C$ of that experiment is higher than in either Version $\mathrm{C}$ of $\mathrm{Ex}-$ periment 4 or Experiment 2.

Earlier we suggested an alternative explanation for Experiment 1 based on increased perceptual difficulty due to changes in color at each location. If color changes did make it more difficult to identify the target digits, performance should have been lower in the alternating condition of Experiment 4, which had the same color differences at each location. Because performance was equivalent between same and alternating conditions in Versions B and C of Experiment 4, any explanation based on color differences seems unlikely.

\section{GENERAL DISCUSSION}

Two important conclusions arise from this series of experiments. The first concerns how selection is accomplished in the visual system, and the second con- 
cerns why selection is necessary. The first two experiments (especially Experiment 2) demonstrate that when subjects must visually identify target digits defined by color in the presence of distractor digits of a different color, the task is more difficult when the target digits appear at different locations in the visual field. This location effect occurs despite the fact that the relative location of the two (or more) targets is not relevant to the correct response; the response will be the same wherever the targets appear. Subjects in this task apparently treat one location preferentially over another. Once a particular location has been selected for this preference or activation, it is easier to process new stimuli that appear at the same location than to shift it to a different location.

It is not possible to tell whether the selection in these experiments was based on other visual properties in addition to location. For instance, for subjects attending to red, processing of only red stimuli might have been facilitated at the selected location, or perhaps only the detection of shape features used to distinguish digits was facilitated at the selected location. Whatever attentional excitation or inhibition is occurring in these tasks, however, at least some of it is location specific. Other experiments (Cave, 1994) have shown that attention to a colored digit such as those used in these displays can speed the detection of another stimulus at the selected location with a different color, shape, and size. Thus the location-based selection seems to be very general.

Another plausible explanation for these results does not include selection by location. ${ }^{3}$ Subjects might have stored as many digits as possible along with their locations into short-term memory, using selection directly by color to increase the chances that target digits were stored. When the distractors were digits (Experiments 1 and 2), they then had to use information about color that was also stored in short-term memory to determine which digits were targets. Matching color with digits was easier when both digits at one location were of the same color, leading to fewer errors when both targets were at the same location. While this account is consistent with these experiments, it does not explain results from Cave (1994). In those experiments, attention to a digit in the presence of a distractor digit of another color speeded the response time to a small dot appearing at the target digit's location. Apparently the spatial attention allocated to a location for the target digit enhanced detection of a very different type of stimulus (the dot) that appeared at the same location. Thus selection by location can account for both results.

The account of visual selection operating according to location is consistent with many other current accounts of visual attention, including "spotlight theories." The experiments described here, though, are among the few that demonstrate selection by location under circumstances in which location is not an explicit part of the task or the instructions. The fact that it occurs under these circumstances underscores the importance of location in the organization of visual information.
Figure 8 gives a rough sketch of how a location-based selection mechanism might work. (This conception is similar to that described in Treisman, 1986 and 1988, and Treisman \& Gormican, 1988.) Processing starts at the bottom, with two "map" representations. The one on the left encodes the presence of the color green, which in this example is the simple visual feature that determines what location should be selected. The second map contains whatever features are used to distinguish characters from one another. The color information, via a general location map, determines which location is selected. Form features from the selected location make their way to the mechanisms that distinguish different characters, while features from the location not selected are blocked. ${ }^{4}$

Experiments 1 and 2 not only demonstrate selection by location, but they provide a tool for measuring when selection by location is used. In Experiments 3 and 4, that tool was used to inquire why this selection mechanism was necessary, and they led to a second conclusion: Although location-based selection occurs when a digit is identified among distractor digits, it does not occur when a digit is identified among letters. If we assume that letter distractors contain many of the same features found in digits, then any feature-level interference among stimuli must not be strong enough to make location-based selection necessary in these experiments. The letter distractors, however, do not pose the same threat of response-level interference as do the digit distractors, because letter distractors (assuming they are accurately identified) will not elicit incorrect digit responses in the way that digit distractors could. If there are letter and digit detectors as in Figure 9, the letter distractors will generally activate only the letter detectors. Because subjects know that the response must be a digit name, the letter detectors will not be allowed to generate responses, and no interference will occur.

Therefore, these data together suggest that locationbased selection is used to ameliorate response-level in-

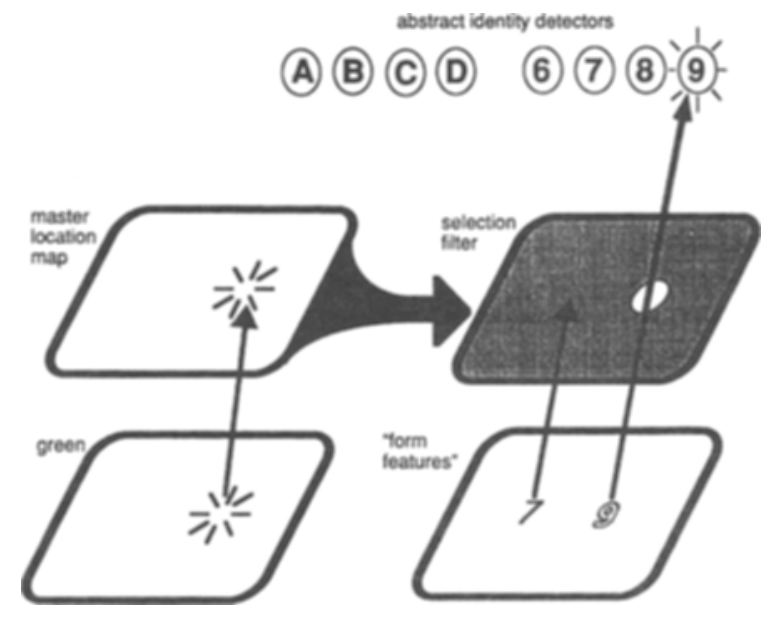

Figure 8. A location-based selection mechanism driven by color information. 
terference in this task, and not the feature-level interference predicted by feature integration theory and guided search. The visual system must be able to detect and combine features efficiently enough to identify two well-spaced characters simultaneously without spatial selection. Feature-level interference may still trigger location-based selection in other tasks with more distractors or with distractors that share more features with the targets. Mozer's (1991) model of recognition illustrates how a system might be able to perform the simple letter discriminations in this experiment without spatial selection, but could still have problems with interference when more distractors are present. Mozer's model also illustrates another important point, that even though location-based selection is used to prevent interference at a high level of processing, the selection itself may work much earlier, at a level below the level of the potential interference. There must also be some ability to gauge the difficulty of perceptual tasks and decide whether spatial selection is necessary.

Styles and Allport (1986) used a different approach to show that selection was necessary to identify characters among distractors of the same category. Their subjects reported letters that appeared in the presence of either other letters or digits. In their experiments, when all the characters in the stimulus array were letters, accuracy was lower for those in the inner positions of the configuration, surrounded by other letters on both sides, and higher for those at one edge of the configuration. However, when subjects were reporting a letter among digits, accuracy did not change with position. Styles and Allport concluded that the disadvantage for inner positions was due to a need for selection, and not to decreased accuracy in feature detection. When the distractors were digits and the target was a letter, selection was not necessary. After performing other experiments with masked stimuli visible for different intervals, they also concluded that the identities of the characters in the display were

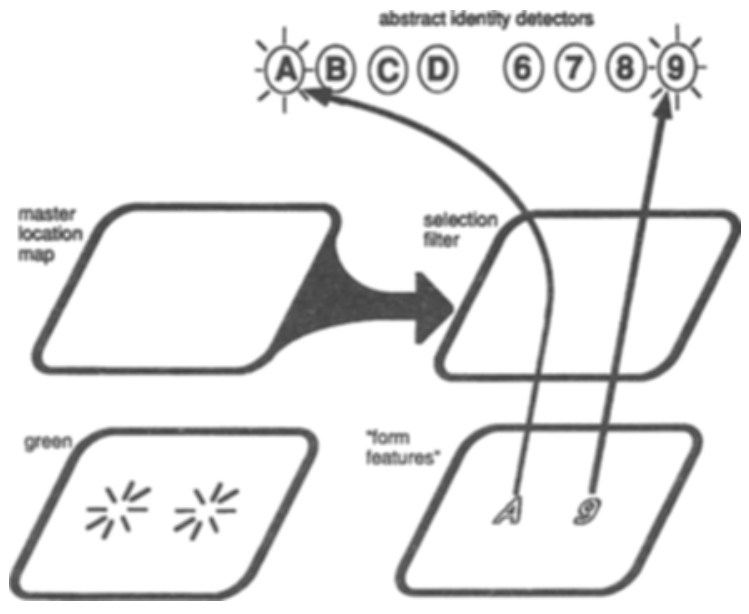

Figure 9. Location-based selection is not used with a digit target and a letter distractor. determined early, but that the identities were not linked with locations or with colors until later in processing.

Of course, selection mechanisms besides the spatial attention demonstrated in our experiments might be (and probably are) at work in different parts of the visual system, and they may be based on properties other than location. In order to disentangle different types of visual selection, we must first learn more about how this location-based mechanism operates and why it is necessary. Elsewhere we have reported tests of whether it is used in tasks other than character identification, whether it is capable of selecting noncontiguous regions, and whether selection allocated for one type of stimulus generalizes to other types (Cave, 1994; Cave \& Pashler, 1994). The most important questions raised here are probably those concerning the conditions under which this type of selection is necessary. Answering these questions will determine when interference occurs in the visual system and will uncover important facts about the general architecture of the visual system.

\section{REFERENCES}

Broadbent, D. E. (1977). Colour, localisation, and perceptual selection. In G. Oléron (Ed.), Psychologie expérimentale et comparée: Hommage da Paul Fraisse (pp. 95-98). Paris: Presses Universitaires de France.

Broadbent, D. E., \& Broadbent, M. H. P. (1987). From detection to identification: Response to multiple targets in rapid serial visual presentation. Perception \& Psychophysics, 42, 105-113.

BUNDESEN, C. (1991). Visual selection of features and objects: Is location special? A reinterpretation of Nissen's (1985) findings. Perception \& Psychophysics, 50, 87-89.

CAVE, K. R. (1994). Measuring the allocation of spatial attention. Manuscript submitted for publication.

CAVE, K. R., \& Kosslyn, S. M. (1989). Varieties of size-specific visual selection. Journal of Experimental Psychology: General, 118, 148164.

CAVE, K. R., \& PASHLer, H. (1994). Visual selection mediated by location: Selecting noncontiguous locations. Manuscript submitted for publication.

CAVE, K. R., \& Wolfe, J. M. (1990). Modeling the role of parallel processing in visual search. Cognitive Psychology, 22, 225-271.

DowniNG, C. J. (1988). Expectancy and visual-spatial attention: Effects on perceptual quality. Journal of Experimental Psychology: Human Perception \& Performance, 14, 188-202.

EGETH, H. (1977). Attention and preattention. In G. H. Bower (Ed.), The psychology of learning and motivation (Vol. 11, pp. 277-320). New York: Academic Press.

ERIKSEN, B. A., \& ERIKSEN, C. W. (1974). Effects of noise letters upon the identification of a target letter in a nonsearch task. Perception \& Psychophysics, 16, 143-149.

ERIKSEN, C. W., \& Hoffman, J. E. (1972). Temporal and spatial characteristics of selective encoding from visual displays. Perception $\&$ Psychophysics, 12, 201-204.

ERIKSEN, C. W., \& Hofrman, J. E. (1973). The extent of processing of noise elements during selective encoding from visual displays. Perception \& Psychophysics, 14, 155-160.

ERIKSEN, C. W., \& HofFMan, J. E. (1974). Selective attention: Noise suppression or signal enhancement? Bulletin of the Psychonomic Society, 4, 587-589.

ERIKSEN, C. W., \& ST. JAMES, J. D. (1986). Visual attention within and around the field of focal attention: A zoom lens model. Perception \& Psychophysics, $40,225-240$.

ERIKSEN, C. W., \& YEH, Y.-Y. (1985). Allocation of attention in the visual field. Journal of Experimental Psychology: Human Perception \& Performance, 11, 583-597. 
Gatti, S. V., \& EgETh, H. E. (1978). Failure of spatial selectivity in vision. Bulletin of the Psychonomic Society, 11, 181-184.

Hoffman, J. E., \& Nelson, B. (1981). Spatial selectivity in visual search. Perception \& Psychophysics, 30, 283-290.

Hoffman, J. E., Nelson, B., \& Houck, M. R. (1983). The role of attentional resources in automatic detection. Cognitive Psychology, $15,379-410$.

LABerge, D. (1983). Spatial extent of attention to letters and words. Journal of Experimental Psychology: Human Perception \& Performance, 9, 371-379.

LABerge, D., \& Brown, V. (1986). Variations in size of the visual field in which targets are presented: An attentional range effect. Perception \& Psychophysics, 40, 188-200.

LABERGE, D., \& BROWN, V. (1989). Theory of attentional operations in shape identification. Psychological Review, 96, 101-124.

LARSEN, A., \& Bundesen, C. (1978). Size scaling in visual pattern recognition. Journal of Experimental Psychology: Human Perception \& Performance, 4, 1-20.

LofTus, G. (1993). Editorial comment. Memory \& Cognition, 21, 1-3.

Mozer, M. C. (1991). The perception of multiple objects. Cambridge, MA: MIT Press.

Murphy, T. D., \& ERIKSEN, C. W. (1987). Temporal changes in the distribution of attention in the visual field in response to precues. Perception \& Psychophysics, 42, 576-586.

NisSEN, M. J. (1985). Accessing features and objects: Is location special? In M. I. Posner \& O. S. M. Marin (Eds.), Attention and performance XI (pp. 205-219). Hillsdale, NJ: Erlbaum.

PODGORNY, R., \& ShEPARD, R. N. (1983). Distribution of visual attention over space. Journal of Experimental Psychology: Human Perception \& Performance, 9, 380-393.

Posner, M. I., Nissen, M. J., \& Ogden, W. C. (1978), Attended and unattended processing modes: The role of set for spatial location. In H. J. Pick \& I. J. Saltzman (Eds.), Modes of perception (pp. 137157). Hillsdale, NJ: Erlbaum.

Posner, M. I., SNyder, C. R. R., \& Davidson, B. J. (1980). Attention and the detection of signals. Journal of Experimental Psychology: General, 109, 160-174.

RAYMOND, J. E., ShaPIRo, K. L., \& ARnell, K. M. (1992). Temporary suppression of visual processing in an RSVP task: An attentional blink? Journal of Experimental Psychology: Human Perception \& Performance, 18, 849-860.

Remington, R., \& Pierce, L. (1984). Moving attention: Evidence for time-invariant shifts of visual selective attention. Perception \& Psychophysics, 35, 393-399.

SAGI, D., \& JULESZ, B. (1985). Fast noninertial shifts of attention. Spatial Vision, 1, 141-149.

SCHNEIDER, W., \& SHIFFrin, R. M. (1977). Controlled and automatic human information processing: I. Detection, search, and attention. Psychological Review, 85, 1-66.

SHIU, L., \& PASHLER, H. (1994). Negligible effect of spatial precuing on identification of single digits. Journal of Experimental Psychology: Human Perception \& Performance, 20, 1037-1054.

Shulman, G. L., Remington, R. W., \& McLean, J. P. (1979). Moving attention through physical space. Journal of Experimental Psychology: Human Perception \& Performance, 5, 522-526.

SNYDER, C. R. R. (1972). Selection, inspection, and naming in visual search. Journal of Experimental Psychology, 92, 428-431.

STYles, E. A., \& AllPORT, D. A. (1986). Perceptual integration of identity, location, and colour. Psychological Research, 48, 189-200.

Treisman, A. M. (1986, November). Features and objects in visual processing. Scientific American, 255(5), 114B-126.

Treisman, A. M. (1988). Features and objects: The Fourteenth Bartlett Memorial Lecture. Quarterly Journal of Experimental Psychology, 40A, 201-237.

Treisman, A. [M.], \& Gelade, G. (1980). A feature-integration theory of attention. Cognitive Psychology, 12, 97-136.

Treisman, A. [M.], \& Gormican, S. (1988). Feature analysis in early vision: Evidence from search asymmetries. Psychological Review, 95, $15-48$

TSAL, Y. (1983). Movements of attention across the visual field. Journal of Experimental Psychology: Human Perception \& Performance, 9, 523-530.

Tsal, Y., \& Lavie, N. (1988). Attending to color and shape: The special role of location in selective visual processing. Perception \& Psychophysics, 44, 15-21.

Tsal, Y., \& Lavie, N. (1993). Location dominance in attending to color and shape. Journal of Experimental Psychology: Human Perception \& Performance, 19, 131-139.

Wolfe, J. M., Cave, K. R., \& Franzel, S. L. (1989). Guided search: An alternative to the feature integration model of visual search. Journal of Experimental Psychology: Human Perception \& Performance, 15, 419-443.

\section{NOTES}

1. Hoffman and Nelson (1981) used an inconsistent mapping of stimuli to responses, which Schneider and Shiffrin (1977) claimed would require controlled processing. Hoffman et al. (1983) used a consistent mapping, which according to Schneider and Shiffrin should result in automatic processing over time.

2. The length of the error bars on all the graphs was calculated as $\sqrt{M S I / n}$, where MSI is the mean square for the subject $\times$ same/alternating interaction (error term for the same/alternating factor), and $n$ is the number of values contributing to each mean. This procedure was suggested by Loftus (1993).

3. This alternative was pointed out by Claus Bundesen.

4. Our current data lead to no conclusions about the nature of the abstract identity detectors, and the diagrams should not be interpreted as making any such claims. In fact, there may not be a separate mechanism dedicated to each character (see Mozer, 1991). Likewise, the data presented here allow no conclusions about the shape of the selected area (discussed in Cave and Pashler, 1994), or the sharpness of the boundary between the selected and unselected areas.

(Manuscript received July 6, 1993;

revision accepted for publication November $5,1994$. ) 